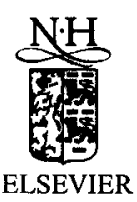

\title{
Electroweak Physics and Ultra High Energy Cosmic Rays
}

\author{
W-Y. P. Hwang ${ }^{a *}$ \\ ${ }^{a}$ Department of Physics, National Taiwan University, \\ Taipei 106, Taiwan, R.O.C.
}

The observation of ultra high energy cosmic rays above the Greisen-Zatsepin-Kuzmin (GZK) cutoff energy has stirred up considerable interest in particle astrophysics and cosmology. In this paper, I wish to summarize, in a sketchy manner, what we have been trying to do recently in connection with this important problem. We believe that the interpretation of these GZK events already requires a full knowledge of the electroweak physics in the energy range of hundreds of $T e V$ 's.

\section{INTRODUCTION}

The observation of ultra high energy cosmic rays (UHECR's) [1-3] above the GreisenZatsepin-Kuzmin (GZK) cutoff energy [4] has stirred up an enormous amount of interest in particle astrophysics and cosmology [5].

The $2.73 \mathrm{~K}$ cosmic microwave background (CMB) is described by Planck's ideal blackbody radiation formula, which yields an average photon number density of 413 photons per $\mathrm{cm}^{3}$ and the mean energy per photon of $6.35 \times 10^{-4} \mathrm{eV}$. A cosmic proton of energy higher than $1.1 \times 10^{20} \mathrm{eV}$, the so-called GZK cutoff energy [4], will interact with the cosmic photons via pion photoproduction reactions, $p+\gamma \rightarrow N+\pi$. Thus, the energy of a cosmic proton, if above GZK cutoff, will degradate and lose its energy until below the GZK cutoff, if a proton could ever be accelerated to such high energy before it propagated towards us through the cosmic medium.

Dimensional analysis [5] suggests that the maximum energy $E$ of a cosmic proton which a cosmic accelerator of mass $M$ with a magnetic field $B$ can ever accelerate is given by

$E \approx \Gamma B M$,

with $\Gamma$ the appropriate Lorentz gamma factor between the cosmic accelerator and us (the observer). For a mass of $10^{9} M_{s u n}$ and $\Gamma \approx 1$, it would call for a magnetic field of larger than $10^{4}$ Gauss in order to obtain a cosmic proton of higher than $10^{20} \mathrm{eV}$. Given the microgauss magnetic field of our own galaxy, no structures are large enough or massive enough to accelerate protons to above GZK cutoff energies. Thus, it is most natural to resort to gigantic black holes, such as active galactic nuclei (AGN) or quasars, as the environment where cosmic rays above the GZK cutoff energies were born. Of course,

\footnotetext{
*This project is supported by the Research Excellence Project on "Cosmology and Particle Astroplysics", as funded by the Ministry of Education of R.O.C., as well as in part by the National Science Council of R.O.C.
} 
a stellar black hole with a mass of about 10 solar mass and a magnetic field of higher than $10^{12}$ Gauss may also deliver cosmic protons above the GZK cutoff energy. The issue would then be whether there would be enough of such high-magnetic-field stellar black holes within our own galaxy. It would not sound like a very plausible scenario, as it would point strongly to pointlike sources for UHECR's.

So, the situation is paradoxical. Our experimental colleagues did observe gigantic showers initiated by some cosmic rays of energies higher than $10^{20} \mathrm{eV}$. It would better be cosmic protons since neither electrons nor photons of such high energies could reach us from outside our own galaxy, but it could not be cosmic protons since it is most unlikely that they could be accelerated to such high energies within our own galaxy and since if accelerated outside our own galaxy such protons would degradate and lose energy till below the GZK cutoff energy.

\section{TRYING OUT WITH NEUTRINOS}

Should we turn to ultra high energy neutrinos as the candidate for UHECR's above the GZK energy?

A neutrino of $10^{20} \mathrm{eV}$ in energy, upon entering the atmosphere or hitting nucleons in the atmosphere, has a $\mathrm{CM}$ energy of $\left(2 E_{\nu} m_{p}\right)^{1 / 2} \approx 450 \mathrm{TeV}$, an energy way beyond any manmade accelerator on earth can ever reach and an energy where no one would doubt that physics beyond the standard model (of electroweak interactions), such as SUSY's, should be properly taken into consideration. In terms of interaction strengths or mean free paths, neutrinos at such energies would not differ much from photons of similar energies. The pre-perception of the neutrinos being only feebly interacting is no longer true. Indeed, a neutrino of $10^{20} \mathrm{eV}$ in energy, upon hitting a nucleon in the atmosphere, would see and interact with a collection of partons, including quarks, antiquarks, and gluons, in a way fairly similar to a photon of the same energy. For instance, if supersymmetric particles, or SUSY's, are indeed there, they may be produced in abundance.

So, what is the characteristics of the showers initiated by ultra high energy neutrinos and how does it differ from those from ultra high energy protons or photons? This is a detailed question which can only be answered through detailed calculations with some specific SUSY models.

If there are enough of cosmic accelerators which can accelerate protons to energies above GZK cutoff energy, then there may be abundance of neutrinos with energies above the GZK cutoff, neutrinos coming out of GZK protons propagating through the cosmic medium. For example, protons may convert to neutrons, via pion photoproduction, and vice versa, and pions decay into neutrinos plus something else. Also a cosmic neutron decays into a proton, an electron, and an antineutrino. So, if GZK protons, i.e., protons with energies greater than the GZK cutoff energy, can be produced everywhere in the universe, say, near AGN's or quasars, it is natural to anticipate that GZK neutrinos are also everywhere and even more abundant - the point is that such neutrinos travel much more freely than GZK protons and there is no need for them to lose the energy to below the GZK cutoff.

Cross sections for weak interactions at low energies behave like $G_{F}^{2} s$, with $G_{F}$ the Fermi coupling constant and $s$ the overall center-of-mass squared. The electromagnetic cross 
section scales like $\alpha / s$. So, at $s \sim \sqrt{\alpha} / G_{F}$ or $\sqrt{s} \sim 100 \mathrm{GeV}$, neutrinos would behave much like photons. At these energies or higher (such as $450 \mathrm{TeV}$ mentioned above), propagation of neutrinos through the atmosphere or the earth cannot be drastically different from that of photons. At low energies, there are upward neutrinos coming from the opposite site of the earth. But this would cease to be the case when the neutrino energy reaches a certain value such that $\sqrt{s} \sim 100 \mathrm{GeV}$ or higher. In other words, the earth or even the atmosphere will become less transparent, and eventually somewhat opaque, when the energy of the cosmic neutrino reaches $10^{14} \mathrm{eV}$ or higher.

To sum up, the scenario for the origin of UIIECR's is a standard one and is perhaps the most natural. Cosmic protons above GZK energies are accelerated locally near AGN's or quasars which are distributed pretty much uniformly over the entire universe. Propagation of such GZK protons produces numerous neutrinos, many of which have energies exceeding the GZK cutoff energy. Granting the abundance of such "secondary" GZK neutrinos and noting that the earth and the atmosphere would no longer be transparent for such neutrinos, most of the observed UHECR's may be attributed to such GZK neutrinos.

In comparison, we wish to bring up the now famous " $Z$-bursts" proposal [6-8]. In this proposal, the observed showers above the GZK cutoff energy are still assumed to be caused by nucleons or antinucleons - such GZK nucleons or antinucleons are decay products of the $Z$-burst which is caused by some extremely high energy neutrino annihilating with the cosmic background (CB) neutrinos at the center of mass energy $\sqrt{s} \approx M_{Z}$. Such $Z$-bursts need to take place at a distance of at most a few $M p c$ such that the GZK nucleons or antinucleons can reach us without too much degradation in energy due to pion photoproduction. Some rest mass for the CB neutrinos in the range of about $(0.01-0.1) \mathrm{eV}$, compatible with the Super-K neutrino oscillation observations, could help bring down the energy of the extremely high energy neutrinos coming from far away, but still extra-ordinarily high, $10^{23}-10^{24} \mathrm{eV}$. These extremely high energy neutrinos may come from everywhere in the universe, but the fact that it must sit on top of the $Z^{0}$ resonance excludes all neutrinos of "wrong" energies and the additional requirement on the energy being close to the GUT scale, $10^{25} \mathrm{eV}$, makes it very difficult, if not impossible, to get manufactured, and abundantly, at the first place. After all, it now appears to many cosmologists that the very early universe may have never reached the temperature at the GUT scale.

We are not in a position to offer critiques to the $Z$-burst proposal, since the proposal has in fact additional predictions which can eventually be confronted directly with the observations. The critique that, to facilitate the $Z$-burst proposal, it is not easy to understand the origin of the extremely high energy neutrinos and why they could be so abundant may make it less plausible than the standard scenario which we discussed above, but ultimately the judgement call comes from the experiment. In the standard scenario, the issue has to do with the question of how often a neutrino of $\left(10^{20}-10^{21}\right) \mathrm{eV}$ can initiate the shower near the top of the atmosphere, and how deep it needs to penetrate before initiating such shower. As for the $Z$-burst proposal, the central question has to do with the origin of the extremely high energy neutrinos, a neutrino energy at the GUT scale, and yet the flux must be high enough. 


\section{REMARKS}

It is clear that electroweak physics at hundreds of $\mathrm{TeV}$ energies must be formulated and treated quantitatively in order that the origin of UHECR's near or above the GZK cutoff energy can be fully understood. Conversely, the current observation of about a dozen GZK shower events, and many more expected to come in the near future from Pierre Auger Observatory or CHICO (Caltech), already gives birth to experimental electroweak physics at hundreds of $\mathrm{TeV}$ energies. We believe that this will soon become one of the most exciting forefronts in particle physics as well as in particle astrophysics.

\section{REFERENCES}

1. Particle Data Group, "Review of Particle Physics", Phys. Rev. 66 (2002) 010001-1.

2. http://www-akeno.icrr.u-tokyo.ac.jp/AGASA/

3. T. Abu-Zayyad et al. (HiRes Collaboration), astro-ph/0208243, September 2002; T. Abu-Zayyad et al. (HiRes Collaboration), Astropart. Phys. 16 (2001) 1.

4. K. Greisen, Phys. Rev. Lett. 16 (1966) 748; G. T. Zatsepin and V.A. Kuzmin, Pis'ma Zh. Eksp. Tero. Fiz. 4 (1966) 114 [JETP Lett. 4 (1966) 78].

5. For excellent recent reviews, see, e.g., F. Halzen, astro-ph/0111059; S. Sarkar, hepph/0202013; N. Nagano and A.A. Watson, Rev. Mod. Phys. 72 (2000) 689.

6. T. Weiler, Phys. Rev. Lett. 49 (1982) 234; Astrophys. J. 285 (1984) 495; Astropart. Phys. 11 (1999) 303.

7. D. Fargion, B. Mele, and A. Salis, Astrophys. J. 517 (1999) 725.

8. G. Gelmini and G. Varieschi, Phys. Rev. Lett. 82 (1999) 5202; hep-ph/0201273. 